\title{
Mental disorders and cause-specific mortality ${ }^{\dagger}$
}

\author{
MATTI JOUKAMAA, MARKKU HELIÖVAARA, PAUL KNEKT, ARPO AROMAA, \\ RAIMO RAITASALO and VILLE LEHTINEN
}

\author{
Background The impact of clinically \\ diagnosed mental disorders on mortality \\ in the general population has not been \\ established.
}

\begin{abstract}
Aims To examine mental disorders for their prediction of cause-specific mortality.
\end{abstract}

\begin{abstract}
Method Mental disorders were determined using the 36 -item version of the General Health Questionnaire and the Present State Examination in a nationally representative sample of 8000 adult Finns.
\end{abstract}

Results During the 17-year follow-up period 1597 deaths occurred. The presence of a mental disorder detected at baseline was associated with an elevated mortality rate. The relative risk in men was I.6 (95\% confidence interval I.3-1.8) and in women, I.4 (95\% Cl I.2-1.6). In men and women with schizophrenia the relative risks of death during the follow-up period were $3.3(95 \% \mathrm{Cl} 2.3-4.9)$ and $2.3(95 \% \mathrm{Cl}$ 1.3-3.8) respectively, compared with the rest of the sample. In both men and women with schizophrenia the risk of dying of respiratory disease was increased, but the risk of dying of cardiovascular disease was increased only in men with neurotic depression.

\section{Conclusions Schizophrenia and} depression are associated with an elevated risk of natural and unnatural deaths.

Declaration of interest This study was funded by the Social Insurance Institution of Finland.

†See editorial, pp. 477-478, this issue.
The risk of premature death among psychiatric patients is higher than in the general population (Amaddeo et al, 1995; Sohlman \& Lehtinen, 1999). Only few studies have dealt with the issue of mortality and diagnosed mental disorders in the community. A psychiatric diagnosis per se increases the risk of dying prematurely (Rorsman et al, 1982; Lehtinen et al, 1993). Among men, alcohol-related disorders (Ojesjo et al, 1998) and anxiety or panic disorder (Grasbeck et al, 1996) are associated with excessive mortality. Our own earlier studies have shown that neurotic depression is associated with an elevated risk of death due to cardiovascular disease (Aromaa et al, 1994). As far as we know, no population study exists on cause-specific mortality related to mental disorders. Our aim was to establish the significance of mental disorders as predictors for disease-specific mortality, using a representative population sample and a 17-year follow-up period.

\section{METHOD}

\section{Design}

This study forms a part of the Mini-Finland Health Survey, an extensive epidemiological study of the adult Finnish population. The aim of the study is to measure the health status, morbidity and health needs of Finns aged 30 years or over. The illnesses chosen as the main targets were those most frequently causing working disability: diseases of the cardiovascular and respiratory systems, mental disorders, and diseases of the musculoskeletal system and connective tissue. Baseline examinations took place during the period 1977 1980 and the results have been described in detail elsewhere (Aromaa et al, 1989).

\section{Sample}

The study population was a stratified two-stage cluster sample drawn from the population register to represent Finnish adults aged 30 years and over (Aromaa et al, 1989). In the first stage, 40 representative areas were selected. In the second stage, a systematic sample of inhabitants was drawn from each area. The total sample consisted of 8000 persons (3637 men and 4363 women) from 69 municipalities. The data were collected between 1977 and 1980. The examinations were carried out by the mobile clinic of the Social Insurance Institution (SII) in two phases: a screening phase and a diagnostic (clinical) phase. A total of 7217 persons $(90.2 \%$ of the sample) participated in the screening phase. Regarding the distributions of gender, age and level of education, the participants corresponded closely to the whole Finnish population (Aromaa et al, 1989).

\section{Instruments}

Baseline examination

The methods used and the basic results concerning mental disorders in the MiniFinland Health Survey have been described in detail elsewhere (Lehtinen et al, $1990 a, b)$. The screening for mental disorders was designed to be as sensitive as possible in order to minimise the number of false-negative cases, as no person screening negative was invited to join the clinical phase of the study. The most important part of the screen was the 36-item version of the General Health Questionnaire (GHQ-36; Goldberg, 1972). People were also invited to participate in the clinical phase if SII records indicated that they were receiving a disability pension because of a mental disorder, or were entitled to reimbursable medication for such disorders (according to the National Health Insurance scheme in Finland, all psychoses entitle the individual to reimbursable medication). Subjects were also included if they reported having used health services (including those provided by a general practitioner) for a mental disorder or there was a self-perceived mental disturbance. A total of $35 \%$ of the participants in the screening phase were picked out by various screening instruments; $95 \%$ of them cooperated when invited to participate in the clinical phase of the health examination.

The most important method of psychiatric case identification in the clinical phase was the short version of the ninth edition of the Present State Examination (PSE; Wing et al, 1974), administered by a specialist psychiatric nurse. The training for the use 
of the PSE interview was obtained from the developers of the method. The diagnoses were generated by the computer program CATEGO-ID (Wing et al, 1978). Diagnostic information was also obtained from the following sources: diagnostic data on patients hospitalised for psychiatric care; data recorded by the SII on disability pensions granted because of a mental disorder; data recorded by the SII on the presence of mental disorders (psychoses) entitling the person to reimbursable medication; possible diagnoses of a mental disorder by the physician in the clinical phase of the survey; and diagnostic assessment made by the PSE interviewer. A panel of two psychiatrists went through the mental hospital case notes and psychiatric documents entitling the patient to SII benefits (disability pensions and reimbursable medicines), and checked the validity of the diagnoses. For the purpose of the final diagnostic assessment, all the information collected from the various sources was combined, taking into account its degree of certainty, and eliminating mutually incompatible diagnostic combinations.

In this study the following diagnostic categories were considered: any mental disorder; functional psychoses (especially schizophrenia); mood disorders (neurotic depression and affective psychosis); and all neuroses (anxiety neurosis, phobic neurosis, obsessive neurosis, neurotic depression and other neurosis).

\section{Follow-up}

The mortality of the survey participants has been systematically followed ever since the baseline examination. This information was obtained from the Central Statistical Office of Finland, and the principal causes of death were coded according to the eighth revision of the International Classification of Diseases (World Health Organization, 1974). This report covers the deaths occurring between 1978 and 1994, and represents an observation period of 15-17 years and an overall experience of 80089 person years (men, 39804 person years; women, 49285 person years). During the follow-up period 1597 deaths occurred, of which 876 were caused by any cardiovascular disease (537 by coronary disease, 192 by cerebrovascular disease), 130 by respiratory disease, 341 by different kinds of cancers, 72 by injuries and 20 by suicides.

\section{Statistical analyses}

Cox's proportional hazards regression model (Cox, 1972; Kalbfleisch \& Prentice, 1980) was used to estimate the association between mental disorders and different types of mortality. Age-adjusted relative risks (RR) and their $95 \%$ confidence intervals (CI) were estimated based on this model. Interactions between gender and mental disorders were tested by entering interaction terms of gender and each disorder into the model in addition to age, gender and the disorder. The statistical significance of covariance terms was evaluated by the likelihood ratio test and expressed as exact $P$ values. The proportional hazards assumption was tested by stratification for follow-up time. The SAS software package version 6.12 (SAS Institute, 1997) was used.

\section{RESULTS}

The presence of any mental disorder at the time of the baseline study was associated with a statistically significantly elevated risk of death in men (RR 1.56, 95\% CI $1.32-1.84$, Table 1 ) as well as in women (RR 1.38, 95\% CI 1.18-1.63, Table 2). In men it was due to excess mortality from cardiovascular disease in general, coronary heart disease, respiratory disease, nonsuicidal injuries and suicide. Women with any mental disorder had a higher risk than other women of dying of cardiovascular disease in general, coronary heart disease, respiratory disease and suicide.

Both male and female participants with functional psychoses at the time of the baseline study possessed statistically significantly elevated risks of death. This was especially true in the schizophrenia category. In people with schizophrenia the risk of dying of respiratory disease was almost 10 times that in the other participants. Deaths from respiratory disease in the schizophrenia group were due to chronic bronchitis in three men, bronchial asthma in one woman, and pneumonia in two men and two women. Regarding cardiovascular mortality there seemed to be an interaction with gender and schizophrenia (Tables 1 and 2), although it was not statistically significant $\left(\chi^{2}=2.17, \quad\right.$ d.f. $=1$, $P=0.14$ ). In men the risk of dying was statistically significantly higher in the case of cardiovascular disease in general and especially with regard to coronary disease. In women the risk of dying was higher only in the case of cerebrovascular disease. In men with schizophrenia the risk of death by suicide was also statistically significantly elevated compared with other subjects.

When all the study participants were analysed together and the mortality rates had been adjusted for gender and age, there was a statistically significant association between schizophrenia and death due to cancers (RR 2.13, 95\% CI 1.01-4.51), but this association did not reach statistical significance in either gender alone (Tables 1 and 2).

Men suffering from mood disorders also had an elevated risk of death. In cases of suicide and cardiovascular disease, especially coronary disease, the excess mortality was associated with neurotic depression. Mood disorders in women were associated with an elevated risk of death from injuries and suicide. Neurotic depression was associated with an elevated risk of death from respiratory disease among women. In women, unlike men, neurotic depression did not predict cardiovascular death (Tables 1 and 2). The interaction between gender and mood disorders for the prediction of cardiovascular mortality was statistically significant $\left(\chi^{2}=4.63\right.$, d.f. $=1$, $P=0.03$ ).

To study whether the association between mental disorders and mortality remained during the long follow-up period, we deleted the first 10 years of follow-up from the analysis. Even then, the relative risk of death adjusted for age and gender in subjects with mental disorder was 1.68 (95\% CI 1.37-2.07) compared with those without baseline mental disorder.

\section{DISCUSSION}

Our results show in a representative sample of adult people a clear association between clinically diagnosed mental disorders and mortality, with regard to both natural and unnatural deaths. Having a mental disorder predicted an elevated risk of death from cardiovascular diseases in total, coronary heart disease, respiratory disease and suicide. In particular, schizophrenia and depression are involved. Excess suicidal mortality associated with these disorders, earlier reported on the basis of patient studies, was now confirmed at population level. As far as we know, this is the first comprehensive study on this topic that deals not with patient series, but with 

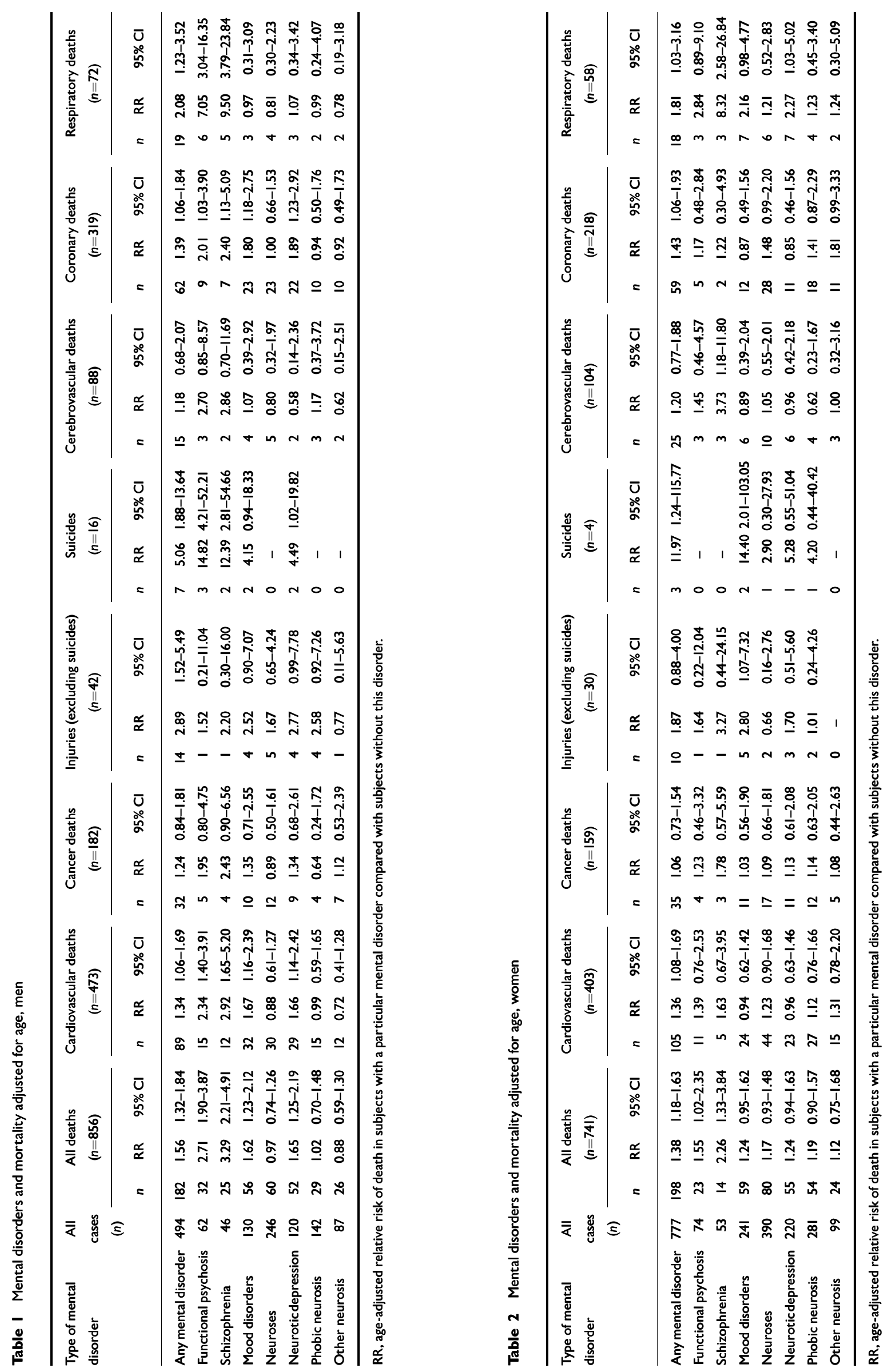
different mental disorders in relation to the general population. A new finding of this study was the association of schizophrenia with mortality from respiratory disease. Our finding of a connection between neurotic depression and premature death due to coronary heart disease in men was in full agreement with our previous study with the same material in a 6-year followup (Aromaa et al, 1994).

\section{Schizophrenia and mortality}

We found a considerable excess mortality from respiratory disease in people with schizophrenia. In a meta-analysis, Brown (1997) reported that natural deaths account for about $59 \%$ of the excess mortality in the schizophrenia group, but there is no clear finding implicating any specific somatic disease. However, according to patient studies the greatest number of excess deaths in schizophrenia are due to infectious diseases and to endocrine, circulatory, respiratory, digestive and genito-urinary disorders (Harris \& Barraclough, 1998). It has been suggested that ordinary and excessive smoking among patients with schizophrenia could elevate lung cancer mortality rates (Masterson \& O'Shea, 1984). Cancer deaths (combined) had only a weak association with schizophrenia in our study. On the other hand, this same Mini-Finland Health Survey material has been used to show that depressed mood was associated with an elevated risk for lung cancer (Knekt et al, 1996). The excess mortality due to respiratory disease, especially chronic bronchitis, is probably associated with smoking. Smoking and other unhealthy habits in people with schizophrenia may also be responsible for the excess mortality from cardiovascular disease.

In earlier studies with patient samples, unnatural causes, suicides and accidents have been the most important reasons for excess mortality in schizophrenia (Brown, 1997; Harris \& Barraclough, 1998). In our study the risk of committing suicide was highly elevated among men. This may be due to the small absolute number of cases of schizophrenia $(n=99)$. In our study no statistically significant association between non-suicidal lethal injuries and schizophrenia was found.

\section{Neurotic depression and mortality}

In our study neurotic depression (in men only) was associated with excess mortality due to coronary heart disease. The relationship between depression and cardiovascular disease has recently come under scrutiny. Pratt et al (1996) suggested that a history of dysphoria and major depressive episode increase the risk of myocardial infarction. The results of this study, as well as those of our earlier study (Aromaa et al, 1994), are in line with this finding. Musselman $e t$ al (1998) have even suggested that treatment of depression in patients with cardiovascular disease increases longevity. Why cardiovascular mortality was associated with mood disorders only in men needs to be investigated further.

We also found an association between suicide and mood disorder. Similar results have been reported in other studies with patient samples (Harris \& Barraclough, 1997, 1998). In this study, non-suicidal lethal injuries also contributed to the excess mortality in cases of neurotic depression in women. Unfortunately, it is not possible to analyse probable suicidal intentions in these cases. It is hoped that future research will confirm our observation.

An interesting finding was that neurotic depression was associated with an elevated risk of dying from respiratory disease in women. Similar findings have been obtained also in patient studies of affective disorders (Newman \& Bland, 1991) and of bipolar disorders (Sharma \& Markar, 1994). Further studies are needed to elucidate the meaning of this association. One possible explanation could be smoking, which is commonly associated with depression (Covey et al, 1998).

\section{Anxiety disorders and mortality}

Neurotic disorders other than depression did not exhibit any association with mortality in this study - a finding that is consistent with previous studies (Rorsman et al, 1982; Murphy et al, 1989; Lehtinen et al, 1993). Much attention has been paid to the possible excess mortality in panic disorder due to cardiovascular disease and suicide (Noyes, 1991). Panic disorder, as a type of anxiety disorder, was added to the diagnostic classifications after our study was completed. The prevalence of panic disorder is low in the normal population (Weissman et al, 1997), and consequently there was likely to be only a small number of subjects with panic disorder in our sample. This may be the reason for the lack of excess mortality among persons with neurotic disturbances, since these disorders were analysed as a single group in our study.

\section{Limitations of the study}

Our sample well represents the entire 30 year-old population of Finland. The large number of subjects made it possible to analyse in detail the associations between mental disorders and deaths due to different somatic diseases. A limitation of the study was the impossibility of using standardised diagnostic criteria for mental disorders. The baseline study was peformed before the era of DSM-III-R or ICD-10 diagnostic classification rules. The main diagnostic tool was the ninth edition of the PSE, a semi-structured standardised psychiatric interview that yields the ICD8 psychiatric diagnoses. It was at that time the foremost and most common psychiatric interview instrument in epidemiological studies in several countries (Wing et al, 1974). In addition to the PSE, diagnostic information was obtained from many other sources. A best-estimate diagnostic procedure (Leckman et al, 1982; Klein et al, 1994) was used. In this study it was not possible to analyse how consistent the diagnoses were during the long follow-up period, and confounding factors relevant to the associations between mental disorder and death were not analysed. Another limitation of the study was that it was not possible to assess the mental health of the participants after the baseline examination. It is highly probable that the group originally classified as free from mental disorder contained some people who subsequently developed a mental disorder. Thus, a cohort study such as this one is likely to provide a conservative estimate of the association between mental disorder and mortality.

\section{REFERENCES}

Amaddeo, F., Bisoffi, G., Bonizzato, P., et al (1995) Mortality among patients with psychiatric illness. A tenyear case register study in an area with a communitybased system of care. British Journal of Psychiatry, 166 783-788.

Aromaa, A., Heliövaara, M., Impivaara, O., et al (1989) Aims, methods and study population. Part I. In The Execution of the Mini-Finland Health Survey (eds A. Aromaa, M. Heliövaara, O. Impivaara, et al). Publication No. ML:88 (in Finnish with English summary). Turku: Social Insurance Institution.

_ , Raitasalo, R., Reunanen, A., et al (1994)

Depression and cardiovascular diseases. Acto

Psychiatrica Scandinavica Supplementum, 377, 77-82.

Brown, S. (1997) Excess mortality of schizophrenia. A meta-analysis. British Journal of Psychiatry, I7I, 502-506. 
Covey, L. S., Glassman, A. H. \& Stetner, F. (1998) Cigarette smoking and major depression. Journal of Addictive Diseases, 17, 35-46.

Cox, D. R. (1972) Regression models and life-tables. Journal of the Royal Statistical Society B, 34, 187-220.

Goldberg, D. P. (1972) The Detection of Psychiatric Illness by Questionnaire. Maudsley Monograph No. 2I. London: Oxford University Press.

Grasbeck, A., Rorsman, B., Hagnell, O., et al (1996) Mortality of anxiety syndromes in a normal population. The Lundby Study. Neuropsychobiology, 33, 118-126.

Harris, E. C. \& Barraclough, B. (1997) Suicide as an outcome for mental disorders. A meta-analysis. British Journal of Psychiatry, I70, 205-228.

_ \& _ (1998) Excess mortality of mental disorder. British Journal of Psychiatry, 173, II-53.

Kalbfleisch, J. D. \& Prentice, R. L. (1980) The Statistical Analysis of Failure Time Data. New York: Wiley

Klein, D. N., Ouimette, P. C., Kelly, K. S., et al (1994) Test-retest reliability of team consensus best-estimate diagnoses of axis I and II disorders in a family study. American Journal of Psychiatry, I5I, 1043-1047.

Knekt, P., Raitasalo, R., Heliövaara, M., et al (1996) Elevated lung cancer risk among persons with depressed mood. American Journal of Epidemiology, 144, 1096-1103.

Leckman, J. F., Sholomskas, D., Thompson, W. D., et al (1982) Best estimate of lifetime psychiatric diagnoses. Archives of General Psychiatry, 39, 879-883.

Lehtinen, V., Joukamaa, M., Jyrkinen, E., et a (1990a) Prevalence of mental disorders among adults in Finland: basic results from the Mini-Finland Health Survey. Acta Psychiatrica Scandinavica, 81 4I8-425.

_, _ _ _ et al (1990b) Need for mental health services of the adult population in Finland: results from the Mini-Finland Health Survey. Acta Psychiatrica Scandinavica, 8I, 426-43I.

_ , Veijola, J., Lindholm, T., et al (1993) Stability and Changes of Mental Health in the Finnish Adult Population. Publication No. AL:36 (in Finnish with English summary). Turku: Social Insurance Institution.

Masterson, E. \& O'Shea, B. (1984) Smoking and malignancy in schizophrenia. British Journal of Psychiatry $145,429-432$.

Murphy, J. M., Monson, R. R., Olivier, D. C., et al (1989) Mortality risk and psychiatric disorders. Results of a general physician survey. Social Psychiatry and Psychiatric Epidemiology, 24, 134-142.

Musselman, D. L., Evans, D. L. \& Nemeroff, C. B. (1998) The relationship of depression to cardiovascular disease: epidemiology, biology, and treatment. Archives of General Psychiatry, 55, 580-592.

Newman, S. C. \& Bland, R. C. (1991) Suicide risk varies by subtype of affective disorder. Acta Psychiatrica Scandinavica, 83, 420-426.

\section{CLINICAL IMPLICATIONS}

Of the mental disorders examined, only schizophrenia and depression increased mortality.

- In people with schizophrenia the risk of dying of respiratory disease is increased.

- Mood disorders in men are associated with excess cardiovascular mortality.

\section{LIMITATIONS}

- A two-stage psychiatric interview was used. It was not possible to interview all subjects.

- Because the basic study was completed in the 1970s it was not possible to use standardised diagnostic criteria for the various mental disorders.

- The sample was not large enough to examine the relative risks for all possible mental disorders associating with mortality.

MATTI JOUKAMAA, Department of Psychiatry, Oulu University Hospital, Oulu; MARKKU HELIÖVAARA, PAUL KNEKT, ARPO AROMAA, National Public Health Institute, Helsinki; RAIMO RAITASALO, Social Insurance Institution, Helsinki; VILLE LEHTINEN, National Research and Development Centre for Welfare and Health, Helsinki, Finland

Correspondence: Matti Joukamaa, Department of Psychiatry, Oulu University Hospital, PO Box 5000 FIN-9040I Oulu, Finland. Tel: +358 8315 7374; fax: +358 8333 167; e-mail: matti.joukamaa@oulu.fi

(First received 28 September 2000, final revision II June 200I, accepted II June 200I)

Noyes, R. (1991) Suicide and panic disorder: a review. Journal of Affective Disorders, 22, I-II.

Ojesjo, L., Hagnell, O. \& Otterbeck, L. (1998) Mortality in alcoholism among men in the Lundby Community Cohort, Sweden: a forty-year follow-up. Journal of Studies on Alcohol, 59, 140-145.

Pratt, L. A., Ford, D. E., Crum, R. M., et al (1996) Depression, psychotropic medication, and risk of myocardial infarction: prospective data from the Baltimore ECA follow-up. Circulation, 94, 3123-3129.

Rorsman, B., Hagnell, O. \& Lanke, J. (1982) Mortality in the Lundby study. Natural death in different forms of mental disorder in a total population investigated during a 25-year period. Neuropsychobiology, 8, 188-197.

SAS Institute (1997) SAS/STAT Software: Changes and Enhancements through Release 6.I2. Cary, NC: SAS Institute.
Sharma, R. \& Markar, H. R. (1994) Mortality in affective disorder. Journal of Affective Disorders, 31, 91-96.

Sohlman, B. \& Lehtinen, V. (1999) Mortality among discharged psychiatric patients in Finland. Acta Psychiatrica Scandinavica, 99, 102-109.

Weissman, M. M., Bland, R. C., Canino, G. J., et a (1997) The cross-national epidemiology of panic disorder. Archives of General Psychiatry, 54, 305-309.

Wing, J. K., Cooper, J. E. \& Sartorius, N. (1974) The Measurement and Classification of Psychiatric Symptoms. London: Cambridge University Press.

_ , Mann, S. A., Leff, J. P., et al (1978) The concept of a case in psychiatric population surveys. Psychological Medicine, 8, 203-217.

World Health Organization (1974) The Eighth Revision of the International Classification of Diseases and Related Health Problems (ICD-8). Geneva: WHO. 